\title{
Descripción del Modelo PNL en alumnos de Telesecundaria mediante métodos aplicativos de minería de datos, usando el algoritmo K means
}

\author{
Description of the NLP Model in Telesecundaria Students Through Application Methods of \\ Data Mining, Using the K Means Algorithm
} Felipe J Núñez-Cárdenas ${ }^{a}$, Santos Hernández-Hernández ${ }^{b}$, Ana M. Felipe-Redondo ${ }^{c}$

\begin{abstract}
:
The present work focuses on the description of learning styles in Telesecundaria students where they were evaluated individually by group and in general, for this description the evaluation instrument called NLP test was used, from which the learning style of the student can be determined. the three modalities handled by the NLP test (Visual, Auditory and Kinesthetic), to obtain knowled ge the WEKA tool was applied by applying the K-Means algorithm, which is used in the clustering technique that allows us to model the description of these groups.
\end{abstract}

Keywords:

Learning styles, WEKA, K-Means, Data mining, NLP test

\section{Resumen:}

El presente trabajo se centra en la descripción de estilos de aprendizaje en alumnos de Telesecundaria donde se evaluaron individualmente por grupo y en general, para esta descripion se utilizo el instrumento de evaluación denominado test de PNL, del cual se puede determinar el estilo de aprendizaje de las tres modalidades que maneja el test PNL (Visual, Auditivo y Kinestésico), para obtener el conocimiento se utilizó la herramienta WEKA aplicando el algoritmo K-Means, el cual se utiliza en la técnica de clustering que nos permite modelar la descripción de estos grupos.

\section{Palabras Clave:}

Estilos de aprendizaje, WEKA, K-Means, Minería de datos., modelo PNL

\section{INTRODUCCIÓN}

Steve Bavister y Amanda Vickers (2014), definen la Programación Neurolingüística como un modelo de comunicación que se centra en identificar y usar modelos de pensamiento que influyan sobre el comportamiento de una persona como una manera de mejorar la calidad y la efectividad de la vida. Un problema del PNL es la naturaleza de su nombre, ya que cuando se menciona el término Programación Neurolingüística a personas que nunca han oído hablar de él, la reacción suele ser un poco negativa. Por otra parte, el nombre podría suscitar que estamos ante técnicas empíricas derivadas de la neurociencia, pero no hay evidencias que constaten la eficacia de estas.

Se realiza este proyecto de minería de datos aplicando el modelo descriptivo, para eso se considera que sería útil detectar las características del perfil de aprendizaje de estudiantes de telesecundaria. Con este propósito se aplico el test a setenta y seis estudiantes de una telesecundaria, utilizando el Test propuesto por Steve y Amanda. Con el fin de descubrir el conocimiento implícito en las respuestas, se siguió el proceso de descubrimiento a través de la técnica de clustering, de minería de datos para detectar el estilo de aprendizaje dominante en los estudiantes de Telesecundaria.

\section{ESTADO DEL ARTE}

Pablo Cazau en su proyecto Estilos de aprendizaje: Generalidades, desde Salvador en el año 2004, da a conocer los modelos de estilos de aprendizaje y la integración de la misma. [1]

En el año 2013, en Argentina, en el proyecto Estilos de aprendizaje de los alumnos de ingeniería, según la programación neurolingüística propuesto por Ana maría 
Tocci, da a conocer como aprenden los estudiantes de la facultad de ingeniería. [2]

En España, en el año 2015 Domingo J. Gallego de la UNED, en su proyecto de investigación llamado "ya he diagnosticado el estilo de aprendizaje de mis alumnos y ahora ¿qué hago?", describe estilos de aprendizajes y su proceso para el análisis del estilo de aprendizaje. [3]

El autor Fernando Maureira de Santiago chile, en el año 2012, en su proyecto estilos de aprendizaje visual, auditivo o kinestésicos de los estudiantes de educación física de la Uisek de Chile, da a conocer en su investigación uno de los estilos de aprendizaje que se maneja en la parte deportiva. [4]

En el año 2012, en el país de México, González en su revista el modelo VAK y el diseño de curso en línea, provee una cuantificación de los estilos de aprendizaje de los estudiantes. [5]

En el año 2006, en el país del Salvador José C. Riquelmel en su artículo Minería de Datos: Conceptos y tendencias da a conocer la minería de datos, su aplicación, sus técnicas y sus retos y tendencias. [6]

El autor Romero Morales Cristóbal de Córdoba en el año 2005 trabaja en un proyecto del estado actual de la aplicación de la minería de datos de los datos del sistema de enseñanza basado en la web. En donde la aplicación específica de técnicas de minería de datos a los sistemas de enseñanza a distancia basados en web, además utiliza las reglas de asociación, secuencia de patrones, clasificación y agrupación. [7]

En el año 2007, Duran E. en su trabajo que tiene por nombre Minería de datos para descubrir estilos de aprendizaje, a partir de la aplicación de tenicas para detectar estilos de aprendizaje dominante en un grupo de estudiantes encuestados de la carrera de Licenciatura en Sistemas de Información (LSI) utilizando el Test propuesto por Felder y Soloman. [8]

En el año $2007 \mathrm{~F}$. Valenga en su proyecto titulado Aplicación de minería de datos para la exploración y detección de patrones delictivos en Argentina, busca analizar los homicidios cometidos en la República de Argentina mediante una herramienta de distribución libre. [9]

El autor I. Perversi de argentina trabajó con el proyecto titulado Identificación y detección de patrones delictivos basados en minería de datos. Donde el objetivo principal fue identificar y detectar los patrones delictivos. Y como herramienta utilizo la clasificación de datos con ID3 o arboles de decisión. [10]

Elena Duran determina un alto grado de homogeneidad en el estilo de aprendizaje de alumnos de la LSI, en su proyecto Minería de datos para descubrir estilos de aprendizaje, mediante el algoritmo Clustering aplicando la herramienta Weka con el modelo PNL en el año 2012. [11]
En el proyecto Diagnostico del estilo de aprendizaje predominante basado en minería de datos y el modelo del felder: aplicaciones al elearning 3.0., se identifica el proceso de diagnóstico del estilo de aprendizaje de un grupo de estudiantes específicos, mediante el algoritmo Clustering con la herramienta Weka utilizando el modelo Felder, realizado por Yasunari del V. Ramirez Leon de España, realizado en el año 2012. [11]

En la ciudad de Huejutla en el año 2013, el autor Nùñez Càrdenas realiz el proyecto que lleva por nombre Identificación de Estilos de Aprendizaje en Alumnos Universitarios de Computación de la Huasteca Hidalguense mediante Técnicas de Minería de Datos, el cual se centra en la aplicación de técnicas de minería de datos para descubrir las combinaciones de estilos de aprendizaje mostradas por estudiantes de educación superior de computación o áreas afines, en la región de la Huasteca Hidalguense.[17]

\section{FUNDAMENTO TEORICO}

\subsection{TEST DE ESTILO DE APRENDIZAJE PNL}

Este modelo, también llamado visual-auditivo-kinestésico (VAK), toma en cuenta el criterio neurolingüístico que considera que la vía de ingreso de la información (ojo, oído, cuerpo) -o, si se quiere, el sistema de representación (visual, auditivo, kinestésico)- resulta fundamental en las preferencias de quien aprende 0 enseña. Por ejemplo, cuando le presentan a alguien, ¿qué le es más fácil recordar después? ¿la cara (visual), el nombre (auditivo), o la impresión (kinestésico) que la persona le produjo? Más concretamente (Sin mención de autor, 2000a), tenemos tres grandes sistemas para representar mentalmente la información, el visual, el auditivo y el kinestésico. Utilizamos el sistema de representación visual siempre que recordamos imágenes abstractas (como letras y números) y concretas. El sistema de representación auditivo es el que nos permite oír en nuestra mente voces, sonidos, música. Cuando recordamos una melodía o una conversación, o cuando reconocemos la voz de la persona que nos habla por teléfono estamos utilizando el sistema de representación auditivo. Por último, cuando recordamos el sabor de nuestra comida favorita, o lo que sentimos al escuchar una canción estamos utilizando el sistema de representación kinestésico. [13]

\subsubsection{CARACTERISTICAS DE ESTE MODELO}

Sistema de representación visual: Los alumnos visuales aprenden mejor cuando leen 0 ven la 
información de alguna manera. En una conferencia, por ejemplo, preferirán leer las fotocopias o transparencias a seguir la explicación oral, o, en su defecto, tomarán notas para poder tener algo que leer. Cuando pensamos en imágenes (por ejemplo, cuando 'vemos' en nuestra mente la página del libro de texto con la información que necesitamos) podemos traer a la mente mucha información a la vez. Por eso la gente que utiliza el sistema de representación visual tiene más facilidad para absorber grandes cantidades de información con rapidez.

Sistema de representación auditivo: Cuando recordamos utilizando el sistema de representación auditivo lo hacemos de manera secuencial y ordenada. Los alumnos auditivos aprenden mejor cuando reciben las explicaciones oralmente y cuando pueden hablar y explicar esa información a otra persona. En un examen, por ejemplo, el alumno que vea mentalmente la página del libro podrá pasar de un punto a otro sin perder tiempo, porqué está viendo toda la información a la vez. Sin embargo, el alumno auditivo necesita escuchar su grabación mental paso a paso. Los alumnos que memorizan de forma auditiva no pueden olvidarse ni una palabra, porque no saben seguir. Es como cortar la cinta de una cassette. Por el contrario, un alumno visual que se olvida de una palabra no tiene mayores problemas, porqué sigue viendo el resto del texto o de la información. El sistema auditivo no permite relacionar conceptos o elaborar conceptos abstractos con la misma facilidad que el sistema visual y no es tan rápido. Es, sin embargo, fundamental en el aprendizaje de los idiomas, y naturalmente, de la música.

Sistema de representación kinestésico: Cuando procesamos la información asociándola a nuestras sensaciones y movimientos, a nuestro cuerpo, estamos utilizando el sistema de representación kinestésico. Utilizamos este sistema, naturalmente, cuando aprendemos un deporte, pero también para muchas otras actividades. Por ejemplo, muchos profesores comentan que cuando corrigen ejercicios de sus alumnos, notan físicamente si algo está mal o bien. O que las faltas de ortografía les molestan físicamente. Aprender utilizando el sistema kinestésico es lento, mucho más lento que con cualquiera de los otros dos sistemas, el visual y el auditivo. Se necesita más tiempo para aprender a escribir a máquina sin necesidad de pensar en lo que uno está haciendo que para aprenderse de memoria la lista de letras y símbolos que aparecen en el teclado. El aprendizaje kinestésico también es profundo. Nos podemos aprender una lista de palabras y olvidarlas al día siguiente, pero cuando uno aprende a montar en bicicleta, no se olvida nunca. Una vez que sabemos algo con nuestro cuerpo, que lo hemos aprendido con la memoria muscular, es muy difícil que se nos olvide. Los alumnos que utilizan preferentemente el sistema kinestésico necesitan, por tanto, más tiempo que los demás. Decimos de ellos que son lentos. Esa lentitud no tiene nada que ver con la falta de inteligencia, sino con su distinta manera de aprender. Los alumnos kinestésicos aprenden cuando hacen cosas como, por ejemplo, experimentos de laboratorio o proyectos.

\subsection{MINERIA DE DATOS}

El datamining (minería de datos), es el conjunto de técnicas y tecnologías que permiten explorar grandes bases de datos, de manera automática o semiautomática, con el objetivo de encontrar patrones repetitivos, tendencias o reglas que expliquen el comportamiento de los datos en un determinado contexto. Básicamente, el datamining surge para intentar ayudar a comprender el contenido de un repositorio de datos. Con este fin, hace uso de prácticas estadísticas y, en algunos casos, de algoritmos de búsqueda próximos a la Inteligencia Artificial y a las redes neuronales. De forma general, los datos son la materia prima bruta. En el momento que el usuario les atribuye algún significado especial pasan a convertirse en información. Cuando los especialistas elaboran o encuentran un modelo, haciendo que la interpretación que surge entre la información y ese modelo represente un valor agregado, entonces nos referimos al conocimiento. Vea más diferencias entre datos, información y conocimiento. [14]

\subsection{TECNICA DESCRIPTIVA}

Estos modelos se crean automáticamente partiendo del reconocimiento de patrones. En este grupo se incluye las técnicas de clustering y segmentación (que también son técnicas de clasificación en cierto modo), las técnicas de asociación y dependencia, las técnicas de análisis exploratorio de datos y técnicas de reducción de la dimensión (factorial, componentes principales, correspondencias, etc.) y de escalamiento multidimensional. [15]

\subsection{TECNICA CLUSTERING}

Se denomina clustering al "proceso de agrupar un conjunto de objetos físicos o abstractos en clases de objetos similares". Un cluster es una colección de objetos de datos que son similares a otros dentro del mismo cluster y son distintos a los objetos de otros clusters. El clustering es un ejemplo de aprendizaje no supervisado en el cual se usan métodos que intentan encontrar particiones naturales de patrones. 
Se trata de una técnica descriptiva dentro de la Minería de Datos (MD), muy útil para identificar patrones que explican o resumen los datos mediante la exploración de las propiedades de los mismos. [16]

\section{Actividades de análisis de clustering.}

* Representación de patrones. Se refiere al establecimiento del número de clases, número de patrones, y el número, tipo y tamaño de las características disponibles para el algoritmo de clustering.

* Definición de proximidad. La proximidad de los patrones es usualmente medida por una función distancia entre un par de datos.

* Clustering. La etapa de agrupamiento puede desarrollarse en un gran número de formas. Se pueden utilizar agrupamientos de clusters jerárquicos, particionales y otros más que abarcan métodos probabilísticos o de teoría de grafos.

* Abstracción de datos. Es el proceso de extraer una representación simple y compacta del conjunto de datos.

* Verificación de resultados. Consiste en validar el análisis de cluster realizado evaluando los resultados obtenidos.

\subsection{WEKA}

Weka (Entorno Waikato para el Análisis del Conocimiento) es una conocida suite de software escrito en java para máquinas de aprendizaje que soporta varias tareas típicas de minería de datos, especialmente pre procesamiento de datos, agrupamiento, clasificación, regresión, visualización y características de selección. Sus técnicas se basan en la hipótesis de que los datos están disponibles en un único archivo plano o relación, donde cada punto marcado es etiquetado por un número fijo de atributos. WEKA proporciona acceso a bases de datos SQL utilizando conectividad de bases de datos Java y puede procesar el resultado devuelto como una consulta de base de datos. Su interfaz de usuario principal es el Explorer, pero la misma funcionalidad puede ser accedida desde la línea de comandos o a través de la interfaz de flujo de conocimientos basada en componentes. [18]

\section{METODOLOGÍA}

\subsection{METODOLOGIA CRISP-DM}

La metodología CRISP-DM, que son siglas de CrossIndustry Standard Process for Data Mining, es un método probado para orientar sus trabajos de minería de datos. [16]

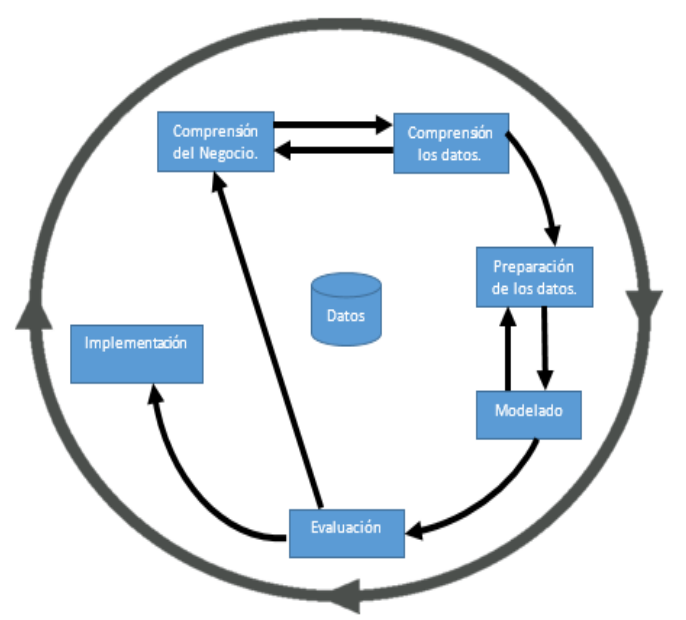

Ilustración 1 Metodología CRISP-DM

\section{COMPRENSIÓN DE NEGOCIO}

En la fase de Entendimiento del Negocio se deben entender los objetivos del proyecto y los requerimientos desde una perspectiva del negocio y luego convertir este conocimiento en una definición de un problema de explotación de información y diseñar un plan preliminar para lograr dichos objetivos.

\section{ENTENDIMIENTO DE DATOS}

El Entendimiento de los Datos comienza con la recolección inicial de datos y procede con las acciones para familiarizarse con ellos, identificar problemas de calidad, identificar primeras pautas en los datos 0 detectar subconjuntos interesantes de las hipótesis de información oculta.

\section{PREPARACIÓN DE LOS DATOS}

La fase de Preparación de los Datos cubre todas las actividades para construir el conjunto de datos final desde los datos iniciales, las tareas de esta fase pueden ser realizadas muchas veces y sin un orden preestablecido, incluye tanto la selección de tablas, registros y atributos como transformación y limpieza de datos para herramientas de modelado.

\section{CONSTRUCCIÓN DEL MODELO}

El Modelado incluye la selección de técnicas de modelado y la calibración de sus parámetros a los valores óptimos, suelen existir distintas técnicas para un mismo 
problema de explotación de información y cada una de ellas suele tener ciertos requisitos sobre los datos, muchas veces es necesario volver a la fase de preparación de los datos.

\section{EVALUACIÓN}

La Evaluación requiere la construcción de uno o varios modelos que aparentan tener la mayor calidad desde una perspectiva de análisis, requiere la evaluación del modelo y revisión de los pasos ejecutados para la construcción del modelo para asegurarnos de lograr los objetivos de negocio, al final de esta fase se debería poder tomar una decisión respecto de la utilización de los resultados.

\section{DESPLIEGUE}

La fase de despliegue puede ser tan simple como generar un reporte o tan compleja como implementar un proceso de explotación de información repetible a través de toda la empresa.

\section{DESARROLLO}

A continuacion se detalla el Desarrollo del Proyecto de acuerdo a las fases de la Metodolgia antes descrita.

\section{COMPRENSIÓN DEL NEGOCIO}

En esta primera etapa de la metodología CRIPS-DM se consideró como población para el caso de este proyecto a todos los alumnos de la Telesecundaria de Panacaxtlan Huejutla Hgo. El instrumento se aplicó a alumnos de $1^{\circ}$, $2^{\circ}$ y $3^{\circ}$ grado, equivalente a 76 alumnos.

El instrumento para la recolección de la información está conformado por 40 reactivos con tres opciones cada una, en donde cada respuesta representa cada uno de las modalidades de estilo de aprendizaje VAK.

\begin{tabular}{|c|c|}
\hline \multicolumn{2}{|c|}{$\begin{array}{c}\text { TEST ESTILO DE APRENDIZAJE (MODELO PNL) } \\
\text { INSTRUCCIONES: Elige una opción con la que más te identifiques de cada una de las preguntas. }\end{array}$} \\
\hline 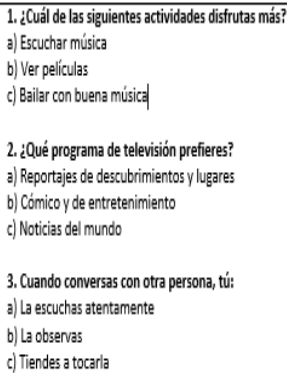 & 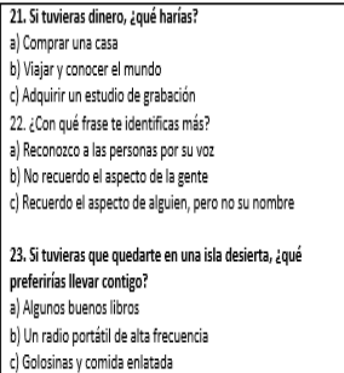 \\
\hline
\end{tabular}

Ilustración 2 Estructura de reactivos de Cuestionario $P N L$
En esta segunda etapa se realiza la recolección de los datos, referentes a los alumnos de Telesecundaria, de 76 alumnos de $1^{\circ}, 2^{\circ}$ y $3^{\circ}$ grado, del instrumento de estilo de aprendizaje VAK. Por cada instrumento aplicado el resultado es un conjunto de números (vector) que representan las incidencias de los tres estilos de aprendizaje que se desea describir, por consiguiente, se describe la manera de evaluación de cada cuestionario aplicado.

La valoración del test de estilo de aprendizaje.

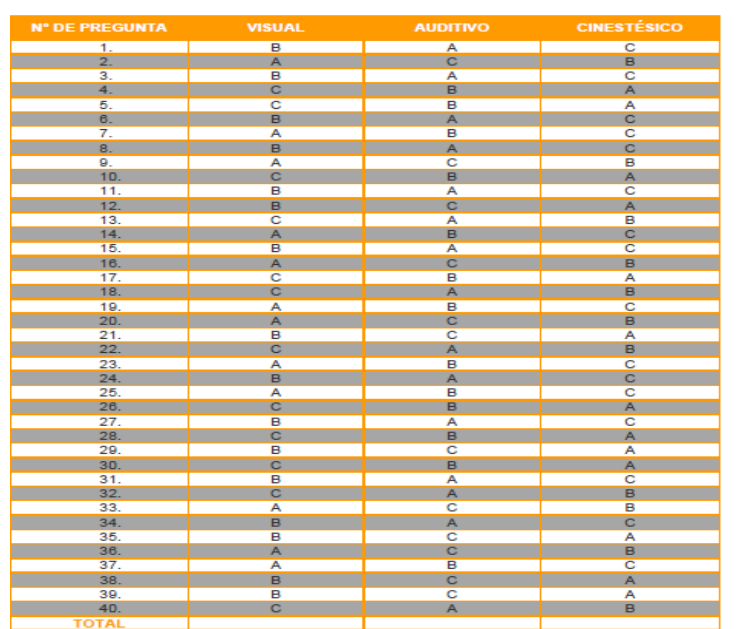

Ilustración 3 Asignación de los valores al cuestionario PNL

El cálculo de la puntuación de cada alumno los valores de cada pregunta

Total de $\mathrm{V}$ marcadas $=\mathrm{R}$

Total de A marcadas $=R$

Total de $\mathrm{K}$ marcadas $=\mathrm{R}$

En cada una de las preguntas se tendrá una respuesta de cada categoría, de la cual corresponde a la sumatoria de cada una de las modalidades marcadas por el alumno.

\section{PREPARACIÓN DE LOS DATOS}

Una vez obtenido los resultados de la aplicación del instrumento, se procede a preparar los datos iniciales para poder ser usados, para eso se utilizó un editor de texto a la aplicación de block de notas. Posteriormente se generaron cuatro archivos. arff para luego utilizarlos en la aplicación WEKA.

Como se muestra en las siguientes ilustraciones de esta manera son los vectores de los datos obtenidos de la tabla de asignación de valores. 


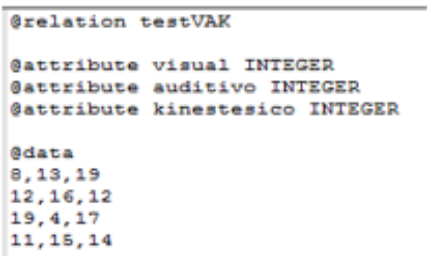

Ilustración 4 Vectores de la encuesta de $1^{\circ}$ grado.

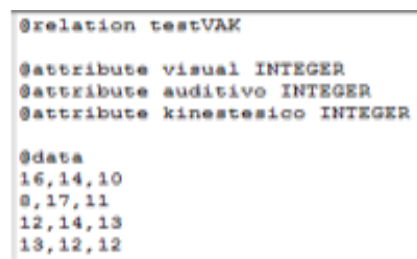

Ilustración 5 Vectores de la encuesta de $2^{\circ}$ grado.

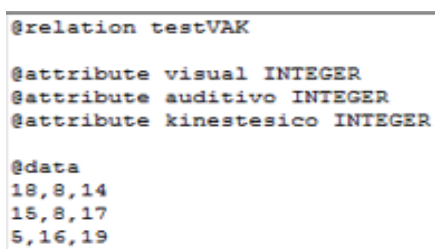

Ilustración 6 Vectores de la encuesta de $3^{\circ}$ grado.

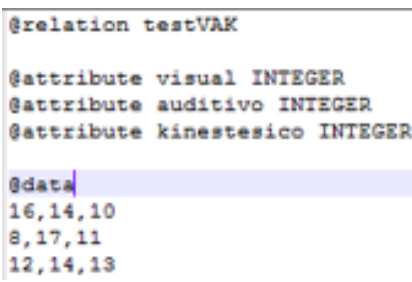

Ilustración 7 Vectores de las encuestas en general.

\section{CONSTRUCCIÓN DEL MODELO}

En esta etapa se seleccionó la técnica descriptiva de Clustering de minería de datos, para ello se vinculo el archivo .arff desde la herramienta Weka.

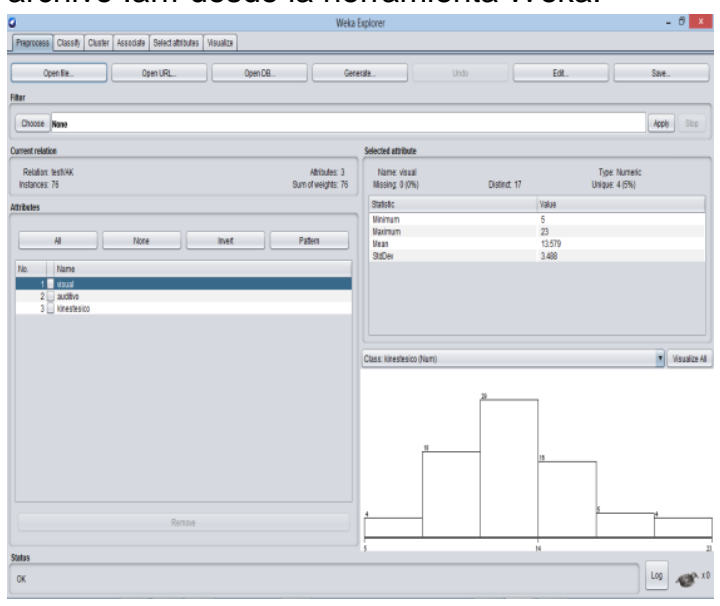

Ilustración 8 Apertura de archivo weka

Para la técnica antes mencionada se utilizó el algoritmo K-Means.
Despues de Analizar los datos, la herramienta WEKA arrojó los correspondientes resultados, con una breve descripción de cada uno de los procesos realizados para la obtención de conocimiento.

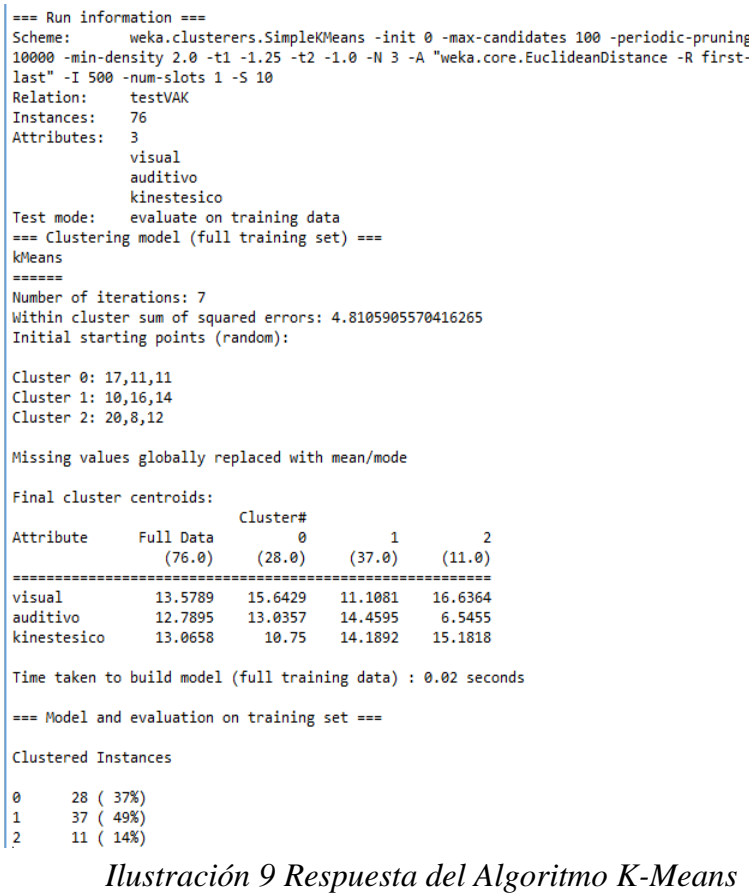

\section{RESULTADOS}

En esta etapa se reflejan los resultados obtenidos, de la aplicación weka, tomando en cuenta, la evaluación y el despliegue de la metodología CRISP-DM. Dando una descripción sobre los datos evaluados.

\section{EVALUACIÓN}

En la construcción del modelo K-Means de los 23 registros que formaba nuestra vista minable del grupo de primer grado, el algoritmo arrojó tres clúster, en el 0 que es representado a la categoría visual se obtuvieron 3 de 23 equivalentes a (13\%), en el auditivo 12 de 23 equivalente a (52\%) y el kinestésico 8 de 23 equivalente (35\%).

\begin{tabular}{|l|l|r|r|r|}
\cline { 3 - 5 } \multicolumn{1}{c|}{} & \multicolumn{3}{c|}{ Cluster\# } \\
\hline Attribute & $\begin{array}{l}\text { Full } \\
\text { Data }\end{array}$ & 0 & 1 & 2 \\
\hline & & 3 & 12 & 8 \\
\hline visual & $13 \%$ & 10.3183 & 12.3023 & 16.104 \\
\hline auditivo & $52 \%$ & 10.3535 & 16.1497 & 10.2027 \\
\hline kinestesico & $35 \%$ & 19 & 11.437 & 13.1005 \\
\hline
\end{tabular}

Ilustración 10 Tabla de resultados del grupo 1

De los 31 registros que formaban nuestra vista minable del grupo de segundo grado, el algoritmo ejecuto tres 
clúster, en el 0 que es representado a la categoría visual se obtuvieron 11 de 31 equivalentes a (35\%), en el auditivo 17 de 31 equivalente a (55\%) y el kinestésico 3 de 31 equivalente (10\%).

\begin{tabular}{|l|l|r|r|r|}
\cline { 3 - 5 } \multicolumn{2}{c|}{} & \multicolumn{3}{c|}{ Cluster\# } \\
\hline Attribute & $\begin{array}{l}\text { Full } \\
\text { Data }\end{array}$ & 0 & 1 & 2 \\
\hline & & 11 & 17 & 3 \\
\hline visual & $35 \%$ & 10.7195 & 16.0371 & 11.1089 \\
\hline auditivo & $55 \%$ & 15.7742 & 12.0182 & 7.1317 \\
\hline kinestesico & $10 \%$ & 12.8213 & 11.6163 & 16.5181 \\
\hline
\end{tabular}

Ilustración 11 Tabla de resultados del grupo 2

De los 22 registros que formaba nuestra vista minable del grupo de tercero, el algoritmo ejecuto tres clúster, en el 0 que es representado a la categoría visual se obtuvieron 15 de 22 equivalentes a (68\%), en el auditivo 3 de 22 equivalente a (14\%) y el kinestésico 4 de 22 equivalente (18\%).

\begin{tabular}{|l|l|r|r|r|}
\cline { 3 - 5 } \multicolumn{2}{c|}{} & \multicolumn{3}{c|}{ Cluster\# } \\
\hline Attribute & $\begin{array}{l}\text { Full } \\
\text { Data }\end{array}$ & 0 & 1 & 2 \\
\hline & & 15 & 3 & 4 \\
\hline visual & $68 \%$ & 11.3455 & 15.9994 & 18.6934 \\
\hline auditivo & $14 \%$ & 14.2609 & 12.6969 & 7.0663 \\
\hline kinestesico & $18 \%$ & 14.3912 & 9.6138 & 14.2351 \\
\hline
\end{tabular}

Ilustración 12 Tabla de resultados del grupo 3

De los 76 registros que formaba nuestra vista minable de los 3 grupo, el algoritmo ejecuto tres clúster, en el cluster 0 que es representado a la categoría visual se obtuvieron 28 de 76 equivalentes a (37\%), en el auditivo 37 de 76 equivalente a ( $49 \%$ ) y el kinestésico 11 de 76 equivalente (14\%).

La evaluación se desarrolló sobre datos de entrenamiento representada en la siguiente tabla.

\begin{tabular}{|l|l|r|r|r|}
\cline { 3 - 5 } \multicolumn{2}{c|}{} & \multicolumn{3}{c|}{ Clúster\# } \\
\hline \multirow{2}{*}{ Attribute } & $\begin{array}{l}\text { Full } \\
\text { Data }\end{array}$ & 0 & 1 & 2 \\
\hline & 76 & 28 & 37 & 11 \\
\hline visual & 13.5789 & 15.6429 & 11.1081 & 16.6364 \\
\hline auditivo & 12.7895 & 13.0357 & 14.4595 & 6.5455 \\
\hline kinestesico & 13.0658 & 10.75 & 14.1892 & 15.1818 \\
\hline
\end{tabular}

Ilustración 13 Evaluación de clúster

\section{DESPLIEGUE}

En etapa de la metodología CRISP-DM se presenta la integración de los diferentes agrupamientos, así como su composición de los diferentes estilos de aprendizaje detectados durante la explotación de los resultados de los cuestionarios aplicados a los alumnos.
En la ilustración 14 como puede observarse que el estilo de aprendizaje domínate en los estudiantes es el Auditivo en el grupo de $1^{\circ}$ grado.

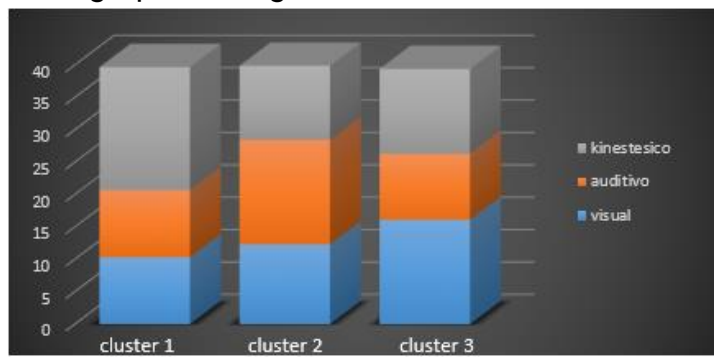

Ilustración 14 Graficación de los datos obtenidos del grupo 1. En la ilustración 15 como puede observarse que el estilo de aprendizaje domínate en los estudiantes es el Kinestesico en el grupo de $2^{\circ}$ grado.

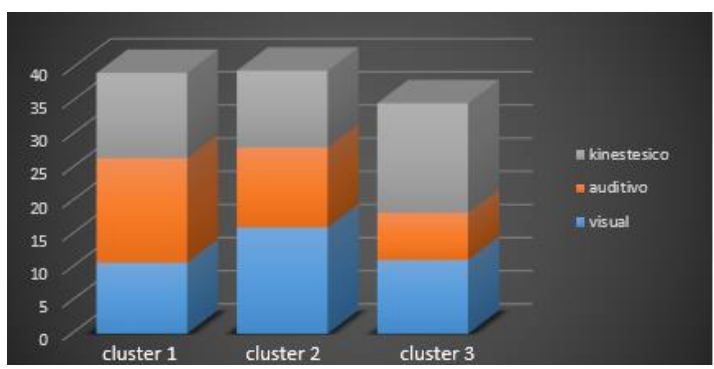

Ilustración 15 Graficación de los datos obtenidos del grupo 2.

En la ilustración 20 como puede observarse que el estilo de aprendizaje domínate en los estudiantes es el Visual en el grupo de $3^{\circ}$ grado.

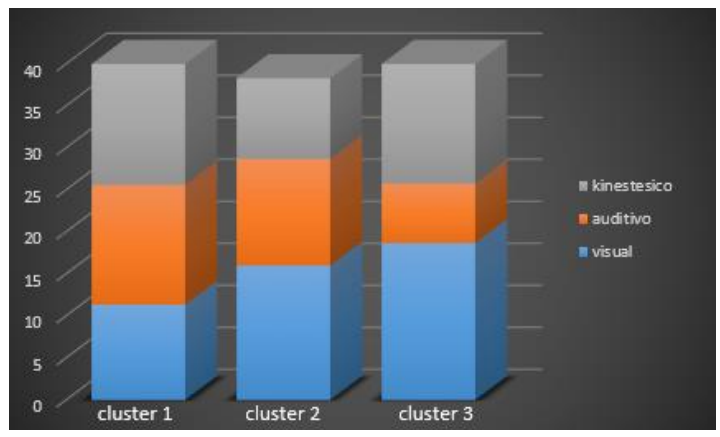

Ilustración 16 Graficación de los datos obtenidos del grupo 3.

En la ilustración 21 como puede observarse que el estilo de aprendizaje domínante en los estudiantes en general es estilo Visual. 


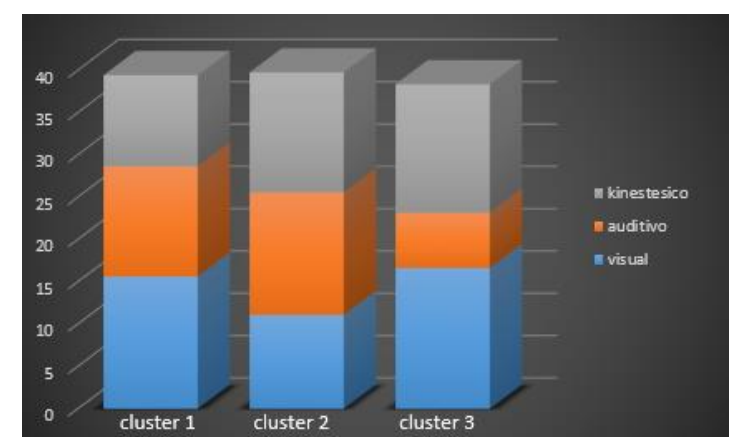

Ilustración 17 Graficación de los clúster obtenidos de datos generales.

\section{CONCLUSIONES Y TRABAJO FUTURO}

El proyecto que se realizó sobre la descripción de los estilos de aprendizaje predominantes en los alumnos de la escuela Telesecundaria de la comunidad de Panacaxtlan, fue de manera favorable durante todo el proceso del presente trabajo.

A partir de los análisis de la información recabada, surgen las siguientes conclusiones:

El estudio de los estilos de aprendizaje de acuerdo a la programación neurolingüística o el sistema VAK, es el que más se ajusta, para la presente investigación, ya que en este nivel (secundaria), es el que permite dar mejor cuenta de la forma en que los alumnos, reciben y procesan la información.

Sobre los estilos de aprendizaje (Visual, Auditivo y Kinestésico) presentados por los alumnos de la escuela telesecundaria, se puede afirmar que están asociados a factores tales como: La metodología de sistema de telesecundaria; los estilos de enseñanza de los docentes, así como las diversas conceptualizaciones que éstos tienen sobre los alumnos, entre otros

El estilo predominante en los alumnos en general, de la escuela telesecundaria, correspondió al sistema visual, destacado por ayudarse de medios o herramientas visuales para adquirir y procesar la información, siendo en este caso la televisión la herramienta fundamental. Y el estilo predominante de cada grupo fue, Auditivo para $1^{\circ}$ grado, Kinestésico para $2^{\circ}$ grado y para $3^{\circ}$ grado el estilo domínante es el visual.

Al hablar de los problemas que pueden presentar los alumnos, para lograr un aprendizaje eficiente, al no estar asociados a ninguna discapacidad visible, se concluye que puede deberse sólo a un estilo de aprendizaje desconocido por docente y no precisamente a un problema.

El conocer sobre los estilos de aprendizaje por parte de los alumnos permitirá reconocer y comprender las diversas formas en que se aprende y se enriquece su manera de relacionarse con el conocimiento.

Se presenta esta investigación y el estudio en el nivel de telesecundaria, porque es el nivel en el cual no existen estudios similares previos, lo cual dará la oportunidad de seguir indagando sobre el tema, ya que aún queda mucho por exponer y concluir.

\section{BIBLIOGRAFÍA}

[1] Cazau, P. (2004). Estilos de aprendizaje: Generalidades. Consultado el, 11(11), 2005

[2] Tocci, A. M. (2013). Estilos de aprendizaje de los alumnos de ingeniería según la programacion neuro lingüística. Journal of learning styles.

[3] Gallego, D. J. (2013). Ya he diagnosticado el estilo de aprendizaje de mis alumnos y ahora ¿qué hago? Journal of Learning Styles.

[4] Maureira Cid, F., Gómez Suazo, A., Flores Ferro, E., \& Aguilera González, J. (2012). Estilos de aprendizaje visual, auditivo o kinestésico de los estudiantes de educación física de la UISEK de Chile. Revista electrónica de psicología Iztacala.

[5] González, B. (2012). El modelo VAK y el diseño de cursos en línea. Revista Mexicana de Bachillerato a Distancia.

[6] Riquelme Santos, J. C., Ruiz, R., \& Gilbert, K. (2006). Mineria de datos: Conceptos y tendencias. Inteligencia artificial: Revista Iberoamericana de Inteligencia Artificial, 10(29)

[7] Romeros Morales, C. (2005). Estado actual de la aplicación de la mineria de datos a los sistemas de enseñanza basado en web. Córdoba.

[8] Durán, E. \&. (2007). Minería de datos para descubrir estilos de aprendizaje.

[9] Valenga, F. B. (2007). Aplicación de Minería de Datos para la exploración y detección de patrones delictivos en Argentina.

[10] Perversi, I. (S.F). Identificación y detección de patrones delictivos basados en Minería de datos. Argentina.

[11] León, Y. D. V. R., \& Carrillo, J. A. O. (2012). Diagnóstico del estilo de aprendizaje predominante basado en minería de datos y el modelo de Felder: aplicaciones al Elearnig 3.0. In Estilos de aprendizaje: investigaciones y experiencias: [V Congreso Mundial de Estilos de Aprendizaje], Santander, 27, 28 y 29 de junio de 2012.

[12] Moreno, B. M. E. H., Imelda, A. M. M., \& Guadalupe, G. R. V. APLICACIÓN DE LA PNL EN ESTILOS DE APRENDIZAJE EN ALUMNOS DE BACHILLERATO.f Yasunari del V. Ramírez León.

[13] Sinnexus. (2016). Sinnexus Bussines Inteligence Informatica estrategica. Obtenido de https://www.sinnexus.com/business_intelligence/datamining.aspx

[14] López, C. P. (2007). Minería de datos: técnicas y herramientas. Editorial Paraninfo.

[15] Farías, R., Durán, E. B., \& Figueroa, S. G. (2008). Las Técnicas de Clustering en la Personalización de Sistemas de e-Learning. In XIV Congreso Argentino de Ciencias de la Computación.

[16] Vanrell, J. Á., \& Bertone, R. A. (2010). Modelo de Proceso de Operación para Proyectos de Explotación de Información. In XVI Congreso Argentino de Ciencias de la Computación.

[17] Nuñez Cárdenas, F. (2013). Identificación de Estilos de Aprendizaje en Alumnos Universitarios de Computación de la Huasteca Hidalguense mediante Técnicas de Minería de Datos.

[18] Vanrell, J. Á., \& Bertone, R. A. (2010). Modelo de Proceso de Operación para Proyectos de Explotación de Información. In XVI Congreso Argentino de Ciencias de la Computación. 